\title{
E2F4 Promotes Neuronal Regeneration and Functional Recovery after Spinal Cord Injury in Zebrafish
}

\begin{abstract}
Shota Sasagawa ${ }^{1 \dagger}$, Yuhei Nishimura ${ }^{1,2,3,4,5 \dagger}$, Yuka Hayakawa ${ }^{1}$, Soichiro Murakami ${ }^{1}$, Yoshifumi Ashikawa ${ }^{1}$, Mizuki Yuge ${ }^{1}$, Shiko Okabe ${ }^{1}$, Koki Kawaguchi ${ }^{1}$, Reiko Kawase ${ }^{1}$ and Toshio Tanaka ${ }^{1,2,3,4,5 *}$

${ }^{1}$ Department of Molecular and Cellular Pharmacology, Pharmacogenomics and Pharmacoinformatics, Mie University Graduate School of Medicine, Tsu, Japan, ${ }^{2}$ Mie University Medical Zebrafish Research Center, Tsu, Japan, ${ }^{3}$ Department of Systems Pharmacology, Mie University Graduate School of Medicine, Tsu, Japan, ${ }^{4}$ Department of Omics Medicine, Mie University Industrial Technology Innovation Institute, Tsu, Japan, ${ }^{5}$ Department of Bioinformatics, Mie University Life Science Research Center, Tsu, Japan
\end{abstract}

\section{OPEN ACCESS}

Edited by:

Trinity Jude Bivalacqua, Johns Hopkins Hospital, USA

Reviewed by:

Medardo Hernández, Universidad Complutense de Madrid,

Spain

Hui Li,

University of Kentucky, USA

*Correspondence:

Toshio Tanaka

tanaka@doc.medic.mie-u.ac.jp

${ }^{\dagger}$ These authors have contributed equally to this work.

Specialty section: This article was submitted to Integrative and Regenerative

Pharmacology,

a section of the journal Frontiers in Pharmacology

Received: 15 January 2016 Accepted: 22 April 2016 Published: 09 May 2016

Citation:

Sasagawa S, Nishimura Y, Hayakawa Y, Murakami S, Ashikawa Y, Yuge M, Okabe S, Kawaguchi K, Kawase $R$ and Tanaka T (2016) E2F4 Promotes Neuronal Regeneration and Functional Recovery after Spinal Cord

Injury in Zebrafish.

Front. Pharmacol. 7:119.

doi: 10.3389/fphar.2016.00119
Mammals exhibit poor recovery after spinal cord injury (SCl), whereas non-mammalian vertebrates exhibit significant spontaneous recovery after SCl. The mechanisms underlying this difference have not been fully elucidated; therefore, the purpose of this study was to investigate these mechanisms. Using comparative transcriptome analysis, we demonstrated that genes related to cell cycle were significantly enriched in the genes specifically dysregulated in zebrafish SCl. Most of the cell cycle-related genes dysregulated in zebrafish SCl were down-regulated, possibly through activation of e2f4. Using a larval zebrafish model of SCl, we demonstrated that the recovery of locomotive function and neuronal regeneration after $\mathrm{SCl}$ were significantly inhibited in zebrafish treated with an E2F4 inhibitor. These results suggest that activation of e2f4 after SCl may be responsible, at least in part, for the significant recovery in zebrafish. This provides novel insight into the lack of recovery after $\mathrm{SCl}$ in mammals and informs potential therapeutic strategies.

Keywords: spinal cord injury, systems pharmacology, comparative transcriptome analysis, zebrafish, E2F4, DREAM complex

\section{INTRODUCTION}

Spinal cord injury (SCI) in mammals typically results in permanent neurological deficits, whereas regenerative organisms, such as amphibians and fish, are capable of regeneration after SCI (reviewed in Lee-Liu et al., 2013; Vajn et al., 2013; Silver et al., 2015). Certain key differences between regenerative and non-regenerative organisms have been identified. For example, in regenerative organisms, astroglial cells form a bridge, and aid axonal elongation after injury. In contrast, after SCI in mammals astrocytes form a glial scar and secrete extracellular matrix (ECM) components such as chondroitin sulfate and proteoglycans. These extrinsic mechanisms can cause a relative lack of growth-promoting molecules and/or a surplus of growth-inhibitory molecules,

Abbreviations: SCI, Spinal cord injury; ECM, extracellular matrix; DEGs, differentially expressed genes; TFs, transcription factors; DREAM complex, TFDP1, RBL2, E2F4, and the MuvB core complex; dpi, days-post-injury; dpf, days-postfertilization. 
which can result in the inhibition of axonal regeneration in mammalian SCI. It has also been demonstrated that the intrinsic propensity for axonal growth is different between regenerative and non-regenerative organisms. For example, the expression of miR-133b was increased at 1 and 7 days after SCI in zebrafish and knockdown of miR-133b significantly attenuated axonal regeneration (Yu et al., 2011). In contrast, the expression of miR$133 \mathrm{~b}$ was transiently increased at $4 \mathrm{~h}$ after SCI and dramatically decreased at 1 day after SCI in rat (Liu et al., 2009). There are several targets of miR-133b that are involved in regeneration after SCI (Vajn et al., 2013). The balance between extrinsic and intrinsic molecular events may determine the overall axonal regeneration response. A comprehensive understanding of the mechanisms underlying the difference between non-regenerative and regenerative organisms is therefore still required.

Transcriptome analysis using microarrays and highthroughput sequencing have provided new insights into the pathogenesis of various disorders, including SCI in mouse (Chen et al., 2013; Wu et al., 2013), rat (Di Giovanni et al., 2003; Chamankhah et al., 2013; Chen et al., 2015), and zebrafish (Ma et al., 2012; Hui et al., 2014). These studies included time-series expression analysis; the clustering of differentially expressed genes (DEGs) based on their temporal expression patterns. This approach has revealed molecular events occurring in the acute, subacute, and chronic stage of SCI. Recently, genome-wide expression profiling was performed to compare the response to SCI in Xenopus laevis at regenerative and non-regenerative stages (Lee-Liu et al., 2014). The analysis revealed that very different sets of transcripts are produced in the regenerative and non-regenerative stages after SCI.

These findings suggest that there might be differences in the DEGs between regenerative and non-regenerative organisms after SCI and that there might be transcription factors (TFs) regulating the DEGs that might be selectively activated or inhibited in either regenerative or non-regenerative organisms. To identify these TFs, we applied systems biology approaches to public transcriptome data for SCI in zebrafish (Hui et al., 2014), mouse (Wu et al., 2013), and rat (Chamankhah et al., 2013). We were able to identify several TFs selectively functioning in zebrafish SCI or mouse/rat SCI. We were also able to demonstrate that e2f4, a member of the DREAM complex (TFDP1, RBL2, E2F4, and MuvB core complex) and a master coordinator of cell cycle dependent gene transcription (Sadasivam and DeCaprio, 2013), was selectively activated in zebrafish SCI and promoted neuronal regeneration and functional recovery.

\section{MATERIALS AND METHODS}

\section{Ethics Statement}

This study was carried out in strict accordance with Japanese law [The Humane Treatment and Management of Animals (2014) ${ }^{1}$, Standards Relating to the Care and Management of Laboratory Animals and Relief of Pain $(2013)^{2}$ and the Guidelines for Proper

\footnotetext{
${ }^{1}$ Ministry of the Environment Japan, 2014, The Law for the Humane Treatment and Management of Animals, Law No. 105, October 1, 1973, revised May 30, 2014.

${ }^{2}$ Ministry of the Environment Japan, Standards Relating to the Care and Management of Laboratory Animals and Relief of Pain, 2013, Notice No. 88, April 28, 2006, revised August 30, 2013.
}

Conduct of Animal Experiments (Science Council of Japan, $2006)^{3}$ ]. All surgery was performed under 2-phenoxyethanol anesthesia, and all efforts were made to minimize suffering.

\section{Compounds}

HLM006474 was obtained from Tocris (Bristol, UK). A stock solution of HLM006474 was prepared by dissolving in dimethyl sulfoxide (Nacalai Tesque, Kyoto, Japan). 2-phenoxyethanol was obtained from Wako Chemical (Osaka, Japan).

\section{Comparative Transcriptome Analysis}

To compare DEGs among mouse, rat, and zebrafish SCI, we used three transcriptome data sets deposited in the Gene Expression Omnibus (GEO; Barrett et al., 2009). In the mouse SCI model (Wu et al., 2013), T9 was injured by contusion with an impactor. In the rat SCI model (Chamankhah et al., 2013), T7 was injured by compression with a clip. In the zebrafish SCI model (Hui et al., 2014), the 15/16th vertebrae was injured by crushing dorso-ventrally with forceps. In these models, the spinal cord containing the epicenter of the injured tissues was extracted for the transcriptome analysis. The raw transcriptome analysis data of mouse SCI (GSE47681) (Wu et al., 2013), rat SCI (GSE45006) (Chamankhah et al., 2013), and zebrafish SCI (GSE39295) (Hui et al., 2014) were downloaded from GEO (Barrett et al., 2009). The raw data were normalized using "affy" (Gautier et al., 2004) for GSE47681 and GSE45006 or "limma" (Ritchie et al., 2015) for GSE39295 in Bioconductor (Gentleman et al., 2004). Probes with reliable signals were selected and subjected to "RankProd" (Hong et al., 2006) to identify DEGs in SCI compared to sham in each model using a false discovery rate of $20 \%$ as the threshold. The gene symbols of DEGs in each model were converted to those of human orthologous genes using Life Science Knowledge Bank (World Fusion, Tokyo, Japan). SwissProt IDs of the human orthologous genes were added using BioMart (Smedley et al., 2015). The list of DEGs is shown in Tables S1, S2 for $1 \mathrm{dpi}$ and 3 dpi, respectively. Venn diagrams of the number of DEGs in these models were drawn using "PINA4MS" (Cowley et al., 2012) in Cytoscape (Shannon et al., 2003).

\section{Identification of Enriched Gene Ontologies in DEGs}

To identify enriched gene ontologies in a given gene list, we used DAVID (Huang Da et al., 2009) with medium classification stringency. The clustering algorithm is based on the hypothesis that similar annotations should have similar gene members. The Group Enrichment Score is the geometric mean (in -log scale) of a member's $p$-values in a corresponding annotation cluster. DEGs specific for zebrafish SCI (Tables S1-8, S2-8), DEGs specific for mouse/rat SCI (Tables S1-9, S2-9), DEGs specific for zebrafish SCI potentially regulated by at least two TFs among e2f4, tfdp1 and foxm1 (Tables S5-1,S5-2), and DEGs specific for mouse/rat SCI potentially regulated by Nfic and Tead4 (Table S5-3,S5-4) were subjected to DAVID. The clusters of enriched gene ontologies for these DEGs are shown in Tables S3, S6. ${ }^{3}$ Science Council of Japan, 2006, Guidelines for Proper Conduct of Animal
Experiments, www.scj.go.jp/ja/info/kohyo/pdf/kohyo-20-k16-2e.pdf 


\section{Identification of Transcription Factors Regulating DEGs Using iRegulon}

iRegulon exploits the fact that genes that are co-regulated by the same TF commonly share binding sites for the TF and uses gene sets derived from ENCODE ChIP-seq data (Gerstein et al., 2012; Janky et al., 2014). We used iRegulon as an application in Cytoscape (Shannon et al., 2003; Nishimura et al., 2015c; Sasagawa et al., 2016). The lists of DEGs specific for zebrafish SCI (Tables S1-8, S2-8) or mouse/rat SCI (Tables S1-9, S2-9) were subjected to iRegulon using the default settings. The predicted transcriptional regulators with normalized enrichment scores (NES) > 3.5 are shown in Table S4.

\section{Zebrafish Strains}

We used an albino zebrafish line (Kelsh et al., 1996) obtained from the Max Planck Institute for Developmental Biology (Tübingen, Germany; Watanabe et al., 2012) for locomotive behavior analysis. We also developed $\operatorname{Tg}$ (eno2: Cerulean) zebrafish. The coding region of Cerulean was amplified by PCR using pCS2+8NCerulean (Addgene, Cambridge, MA, USA) as the template. The PCR product was cloned into Tol2 vector using the In-fusion HD cloning kit (Takara Bio, Shiga, Japan). Briefly, a fragment (from bp 3017 to 1088) of pT2AL200R150G (Kawakami, 2007) was amplified by inverse PCR and fused with the coding region of Cerulean to make a circular plasmid (pT2-cerulean). The promoter of zebrafish eno2 (from -3783 to $3723 \mathrm{bp}$ ) (Bai et al., 2007) was synthesized by Invitrogen (Carlsbad, CA, USA) and cloned into pT2-cerulean using the In-fusion HD cloning kit (Takara Bio) to make pT2eno2-cerulean. pT2-eno2-cerulean and transposase mRNA were injected into zebrafish embryos at the 1-8 cell stage. Larval zebrafish expressing Cerulean or mCherry in the spinal cord were selected and maintained. Mature F0 zebrafish were mated with albino zebrafish. The F1 zebrafish expressing Cerulean were selected and used for in vivo imaging.

Zebrafish were bred and maintained according to previously described methods (Westerfield, 2007; Nishimura et al., 2016). Briefly, zebrafish were raised at $28.5 \pm 0.5^{\circ} \mathrm{C}$ with a $14 \mathrm{~h} / 10$ h light/dark cycle. Embryos were obtained via natural mating and cultured in $0.3 \times$ Danieau's solution [ $19.3 \mathrm{mM} \mathrm{NaCl}, 0.23$ $\mathrm{mM} \mathrm{KCl}, 0.13 \mathrm{mM} \mathrm{MgSO} 4,0.2 \mathrm{mM} \mathrm{Ca}\left(\mathrm{NO}_{3}\right)_{2}, 1.7 \mathrm{mM}$ HEPES, pH 7.2] until $8 \mathrm{dpf}$ for behavioral and in vivo imaging analysis. Zebrafish from $5 \mathrm{dpf}$ were maintained on a diet of live Paramecium.

\section{Microdissection of the Zebrafish Spinal Cord}

Zebrafish were anesthetized with 500 ppm 2-phenoxyethanol in $0.3 \times$ Danieau's solution. The probe (EMB-13Y, Nepa Gene, Chiba, Japan), which was connected to a Gastromaster (GST-1, Nepa Gene) through an adaptor (EMB-C125G, Nepa Gene), was placed on the dorsal skin of a zebrafish above the caudal end of the swim bladder. Then, the foot switch was activated using the "yellow" setting of the Gastromaster until complete transection of the spinal cord was made (Movie S1). After surgery, the zebrafish were transferred to a dish containing $0.3 \times$ Danieau's solution without 2-phenoxyethanol. After about an hour, zebrafish were placed individually into wells of a round 48 -well-plate $(10 \mathrm{~mm}$ diameter, containing $500 \mu \mathrm{L}$ of $0.3 \times$ Danieau's solution). The 48 well-plate was placed in an incubator at $28.5^{\circ} \mathrm{C}$ until the behavior analysis was performed.

\section{Assessment of Locomotion Behavior in Zebrafish after SCI}

An overview of the behavior analysis is shown in Figure 3. All behavioral tests were performed during the same time frame (from 2 to 3 pm at $0,1,2$, and 3 dpi). A 48-well-plate was placed in a DanioVision observation chamber (Noldus, Wageningen, The Netherlands), which was blocked from daylight and illuminated from below with white light $(255 \mathrm{~lx})$ at the time indicated in Figure 4A. The locomotive behavior of zebrafish in each well was monitored by the DanioVision system with a resolution of 1024 $\times 768$ pixels at 25 frames per seconds (fps). After assessment of locomotive behavior at $0 \mathrm{dpi}, 500 \mu \mathrm{L}$ of $0.3 \times$ Danieau's solution with or without $5 \mu \mathrm{M}$ HLM006474 was added to each well of the 48-well-plate and the plate was then placed in an incubator at $28.5^{\circ} \mathrm{C}$ with constant light $(255 \mathrm{~lx})$ from 7 a.m. to 9 p.m. until the behavior analysis was performed the next day. The medium with and without $5 \mu \mathrm{M}$ HLM006474 was changed after the behavior analysis at $0 \mathrm{dpi}$. Two independent experiments were performed.

The recorded video images were analyzed using EthoVision XT11 (Noldus) to measure the locomotive behavior of zebrafish in each well. Mobility was calculated by taking every pixel identified as the subject and comparing it between the current image and the previous one (Nishimura et al., 2015b). If all the pixels were the same, there was zero mobility. If all the pixels were different, there was $100 \%$ mobility. In this study, we defined 5-35, 35-65, and 65-95\% as low, medium, and high mobility. The distance moved by each fish in the dark and light periods was calculated for the time showing medium mobility.

\section{In vivo Imaging of Zebrafish Neurons and Astrocytes at the Lesion Site after SCI}

Zebrafish larvae at $3 \mathrm{dpi}$ with or without HLM006474 treatment were anesthetized with 2-phenoxyethanol (500 ppm in $0.3 \times$ Danieau's solution), then transferred onto glass slides. A few drops of 3\% low-melting agarose solution were placed over the larvae and the larvae were immediately oriented sideways, dorsalside-up. The fluorescent signals in the embedded larvae were observed using an epifluorescent microscope (SMZ25, Nikon, Tokyo, Japan) using CFP filters to detect Cerulean fluorescence. Quantitative assessment of the in vivo fluorescence imaging was performed using Volocity (Perkin Elmer, Cambridge, MA, USA). Briefly, we placed the region of interest (ROI), a circle $191 \mu \mathrm{m}$ in diameter, around the SCI lesion (Figure 5) in the 256-gray-scale (0-255) image. The area of Cerulean fluorescence in the ROI that contained pixels over the intensity threshold (30) was calculated and was used as the fluorescence signal of neurons.

\section{Statistical Analysis}

Statistical analysis was performed using Prism 6 (Graphpad, La Jolla, CA, USA). For the assessment of locomotive behavior, two 
factor ANOVA followed by Sidak's multiple comparison test was performed. For the assessment of neuronal regeneration, Welch's $t$-test was performed. Data are shown as the mean \pm SEM.

\section{RESULTS}

\section{Comparative Transcriptome Analysis Revealed Specific DEGs in Zebrafish SCI and Mouse/Rat SCI}

To identify DEGs that are specific for zebrafish SCI and mouse/rat SCI, we downloaded transcriptome data of mouse SCI (GSE47681) (Wu et al., 2013), rat SCI (GSE45006) (Chamankhah et al., 2013), and zebrafish SCI (GSE39295) (Hui et al., 2014) from the GEO (Barrett et al., 2009). Using these data, we identified DEGs in each SCI model using a false discovery rate of $20 \%$ as the threshold. The identified DEGs and their overlap between the three SCI models are shown in Tables S1, S2 for 1 day-post-injury (dpi) and $3 \mathrm{dpi}$, respectively. Venn diagrams of the numbers of DEGs in these SCI models are shown in Figure 1.

At 1 dpi, 36 DEGs were common among mouse, rat and zebrafish. For 19 of these 36 DEGs, the change in expression was the same in mouse, rat, and zebrafish (Table S1-4), whereas for 17 genes the change in expression was opposite between zebrafish and mouse/rat (Table S1-5). A total of 186 DEGs were identified in zebrafish SCI that were not identified in mouse and/or rat SCI (Table S1-6). Among the 573 DEGs that overlapped between mouse and rat SCI, the change in expression was the same for 553 genes (Table S1-7). In summary, we identified 203 DEGs that were specific for zebrafish SCI (17 DEGs with opposite changes in expression between zebrafish and mouse/rat SCI plus 186 DEGs unique to zebrafish SCI) (Table S1-8) and 570 DEGs that were specific for mouse/rat SCI (17 DEGs with opposite changes in expression between mouse/rat and zebrafish SCI plus 553 DEGs unique to mouse/rat SCI) (Table S1-9).

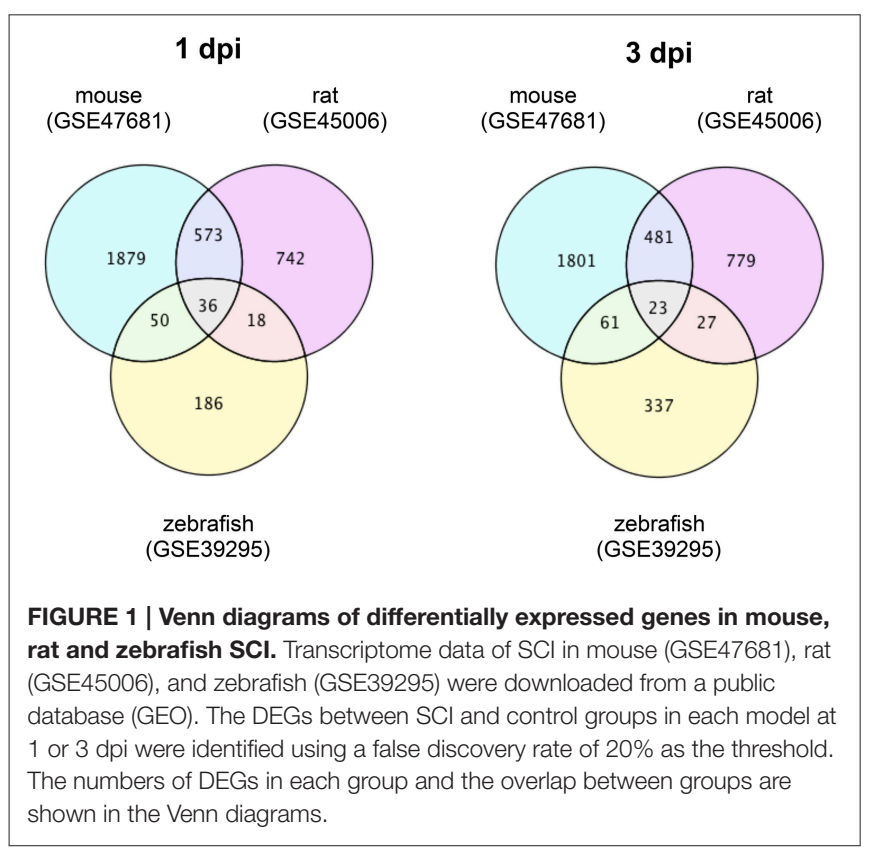

At 3 dpi, 23 DEGs were common among mouse, rat and zebrafish. Of the 23 DEGs, eight had the same change in expression among mouse, rat and zebrafish (Table S2-4), whereas the expression change of 12 genes was opposite between zebrafish and mouse/rat (Table S2-5). The expression changes of three genes were opposite between mouse and rat (data not shown). In zebrafish SCI, 337 DEGs were identified that were not identified in mouse and/or rat SCI (Table S2-6). Among the 481 DEGs that overlapped between mouse and rat SCI, the changes in expression of 419 genes were the same between mouse and rat (Table S2-7). In summary, we identified 349 DEGs specific for zebrafish SCI (12 DEGs with opposite changes in expression between zebrafish and mouse/rat SCI plus 337 DEGs unique to zebrafish SCI) (Table S2-8) and 431 DEGs specific for mouse/rat SCI (12 DEGs with opposite changes in expression between mouse/rat and zebrafish SCI plus 419 DEGs unique to mouse/rat SCI) (Table S2-9).

\section{Genes Related to Cell Cycle Were Enriched in DEGs Specific for Zebrafish SCI}

To reveal the functions of DEGs specific for zebrafish or mouse/rat SCI, we performed gene ontology analysis using DAVID (Huang Da et al., 2009). The analysis revealed that genes related to cell cycle, including mitosis and cyclin, were significantly enriched in DEGs specific for zebrafish SCI at $1 \mathrm{dpi}$ (Table S3-1) and 3 dpi (Table S3-2). Genes related to cell cycle were not enriched in DEGs specific for mouse/rat SCI. Inhibition of cell cycle can stimulate recovery after SCI in mammals (Tian et al., 2007; Wu et al., 2011, 2012). Cell cycle activation after SCI appears to contribute not only to apoptotic cell death of post-mitotic neuronal cells, but also to post-traumatic gliosis and microglial activation (Wu et al., 2011). Therefore, the enrichment of genes related to cell cycle in zebrafish SCI-specific DEGs may be the primary mechanism for neuronal regeneration and functional recovery after SCI in zebrafish, but not in mouse and rat.

\section{Genes Related to Cell Adhesion Were Enriched in DEGs Specific for Mouse/Rat $\mathrm{SCl}$}

We also revealed that genes related to cell adhesion were significantly enriched in DEGs specific for mouse/rat SCI at 1 dpi (Table S3-3) and 3 dpi (Table S3-4). Genes related to cell adhesion were not enriched in DEGs specific for zebrafish SCI. ECM components, such as chondroitin sulfate, proteoglycans and tanascins are known to be up-regulated after injury and to stimulate cell adhesion and contribute to the growth-inhibitory effects of the glial scar (Busch and Silver, 2007). These findings suggest that cell adhesion may be related to the poor recovery from SCI in mammals.

\section{Identification of TFs Regulating Zebrafish SCI-Specific DEGs}

To identify TFs regulating DEGs specific for zebrafish SCI or mouse/rat SCI, we used iRegulon, a bioinformatics analysis tool that has been successfully used to identify TFs for given gene 
lists (Janky et al., 2014; Nishimura et al., 2015c; Sasagawa et al., 2016). The analysis identified four and seven TFs potentially regulating DEGs specific for zebrafish SCI at 1 and $3 \mathrm{dpi}$, respectively (Table S4-1). e2f4, tfdp1, and foxm 1 were common among the DEGs at 1 and $3 \mathrm{dpi}$. Figure $\mathbf{2 A}$ demonstrates the genes that were selectively dysregulated in zebrafish SCI, possibly through e2f4, tfdp1, and foxm1. The genes regulated by at least two of e2f4, tfdp1, and foxm 1 are shown in the inner circle. Most of the genes in the circle were down-regulated (Tables S5-1,S5-2).

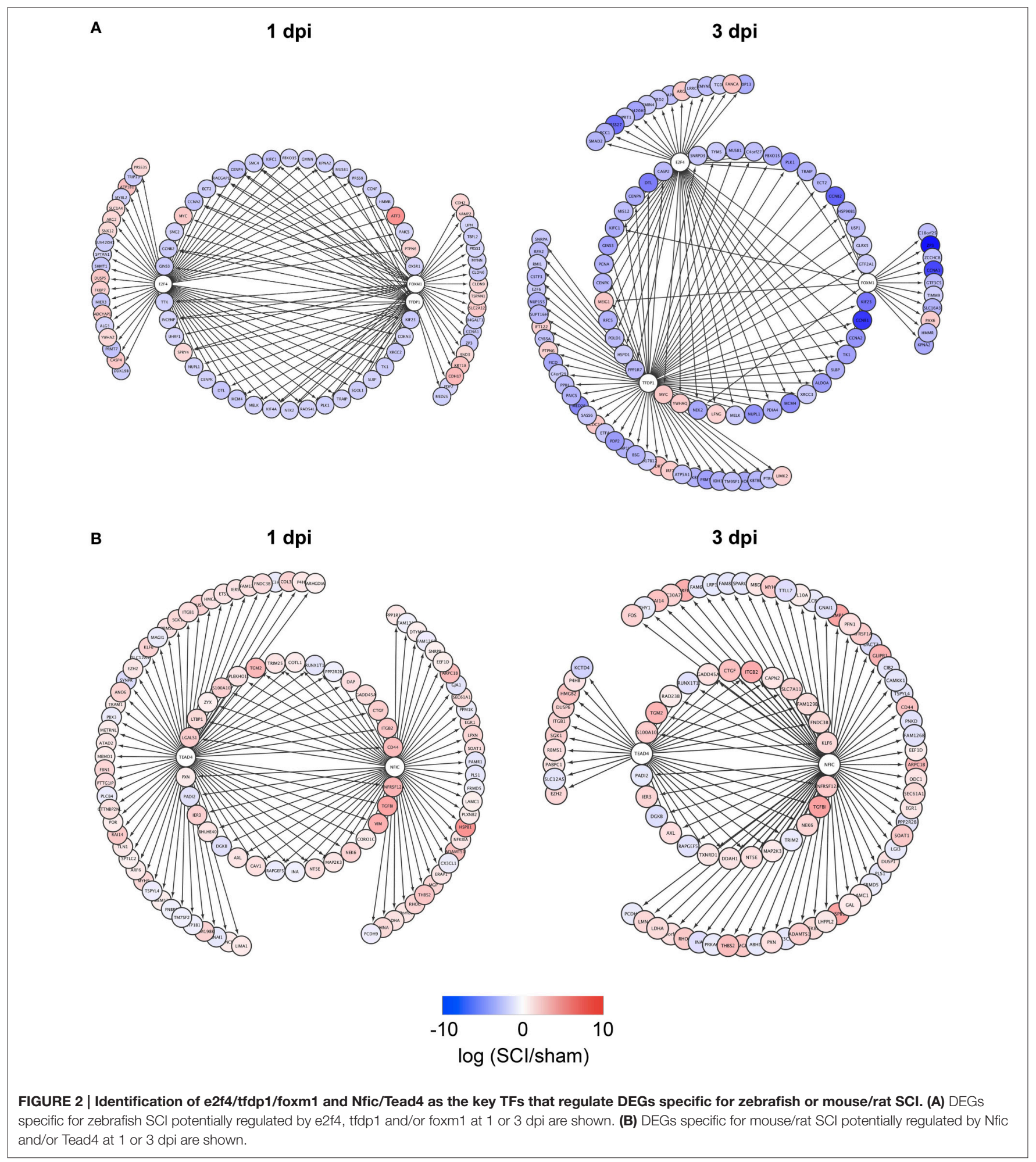




\section{Identification of TFs Regulating Mouse/Rat SCI-Specific DEGs}

We also identified TFs potentially regulating DEGs specific for mouse/rat SCI at 1 and 3 dpi (Table S4-2). Two transcriptional activators Nfic (Pjanic et al., 2011) and Tead4 (Jacquemin et al., 1996) were common between the DEGs at 1 and 3 dpi. The DEGs potentially regulated by Nfic and/or Tead4 are shown in Figure 2B. Most of the DEGs were upregulated in mouse/rat SCI, suggesting that both Nfic and Tead4 may be activated in mouse/rat SCI. Genes related to cell adhesion were significantly enriched in the genes potentially regulated by both Nfic and Tead 4 at 1 but not 3 dpi (Tables S5-3, S6-3 for 1 dpi, Tables S5-4, S6-4 for $3 \mathrm{dpi}$ ). These results suggest that activation of Nfic and Tead4 may contribute to the regulation of DEGs specific for mouse/rat DEG, at least at $1 \mathrm{dpi}$.

\section{The Recovery of Locomotive Behavior after SCI Was Inhibited in Zebrafish Treated with E2F4 Inhibitor}

We wanted to examine whether inhibition of e2f4 would affect the recovery of zebrafish locomotive behavior after SCI. In this study, we developed a novel SCI model using larval zebrafish (Figure 3). Using a microdissection device, complete transection of the spinal cord was made above the caudal end of the swim bladder in zebrafish at 5 days post fertilization (dpf; Movie S1).

To examine the locomotive ability of zebrafish, we utilized the zebrafish behavior of responding to a change in light/dark conditions during short periods (MacPhail et al., 2009; Nishimura et al., 2015a). The experimental conditions are shown in Figure 4A. The locomotion of zebrafish increases greatly when the conditions change from light to dark (Movie S2), whereas the locomotion decreases when the conditions change from dark to light. Additional cycles of alternating light and dark reliably produce alternating levels of low and high locomotion, respectively (MacPhail et al., 2009). The locomotive behavior of zebrafish with SCI treated with or without HLM006474, an inhibitor of E2F4 (Ma et al., 2008), is shown in Figure 4B-D. The recovery of locomotive behavior during the dark periods was significantly lower in zebrafish treated with HLM006474 (Figure 4C). The locomotive behavior during the base and light periods were not significantly different between zebrafish with and without treatment of HLM006474 (Figures 4B,D). The locomotive behavior of control zebrafish was also not affected by HLM006475 treatment during the base, dark or light periods (Figure 4E-G). These results suggest that inhibition of e2f4 attenuates the functional recovery of zebrafish after SCI.

\section{Neuronal Regeneration after SCI Was Attenuated in Zebrafish Treated with E2f4 Inhibitor}

We then examined whether the inhibition of e2f4 might attenuate the neuronal regeneration of spinal cord in zebrafish after SCI. We performed in vivo imaging of zebrafish selectively expressing a fluorescent protein, Cerulean, in neurons under the control of the eno 2 promoter (Figure 5A). As shown in Figures $\mathbf{5 B}, \mathbf{C}$, treatment of HLM006474 significantly reduced the regeneration of neurons at $3 \mathrm{dpi}$. These results suggest that inhibition of e2f 4 attenuates neuronal regeneration after SCI in zebrafish.

\section{DISCUSSION}

\section{Regulation of Zebrafish SCI-Specific DEGs through E2F4, TFDP1, and FOXM1}

In this study, we demonstrated that e2f4, tfdp1, and foxm1 possibly regulate zebrafish SCI-specific DEGs. E2F4 and TFDP1 are members of the DREAM complex, the master coordinator of the cell cycle (Sadasivam and DeCaprio, 2013). The DREAM complex regulates down-regulation of genes related to mitosis
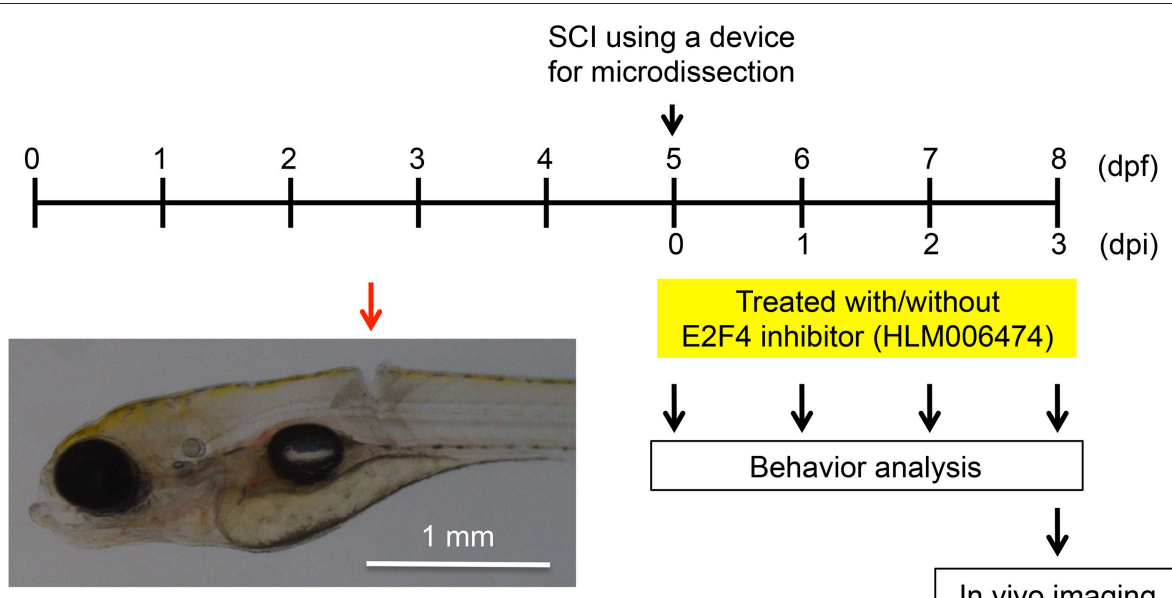

Treated with/without E2F4 inhibitor (HLM006474)

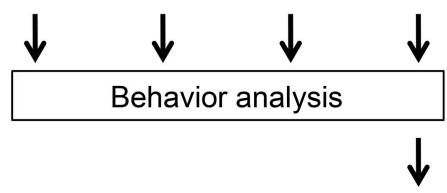

In vivo imaging of spinal cord

FIGURE 3 | Larval zebrafish SCI model used in this study. At 5 dpf, the spinal cords of zebrafish were injured using a device for microdissection (Movie S1). At 0, 1, 2, and 3 dpi, the locomotive behavior of zebrafish was analyzed (Figure 4, Movies S2, S3). At 3 dpi, in vivo imaging of the spinal cord was performed (Figure 5). A representative $\mathrm{SCl}$ in zebrafish is indicated by the red arrow. 
A
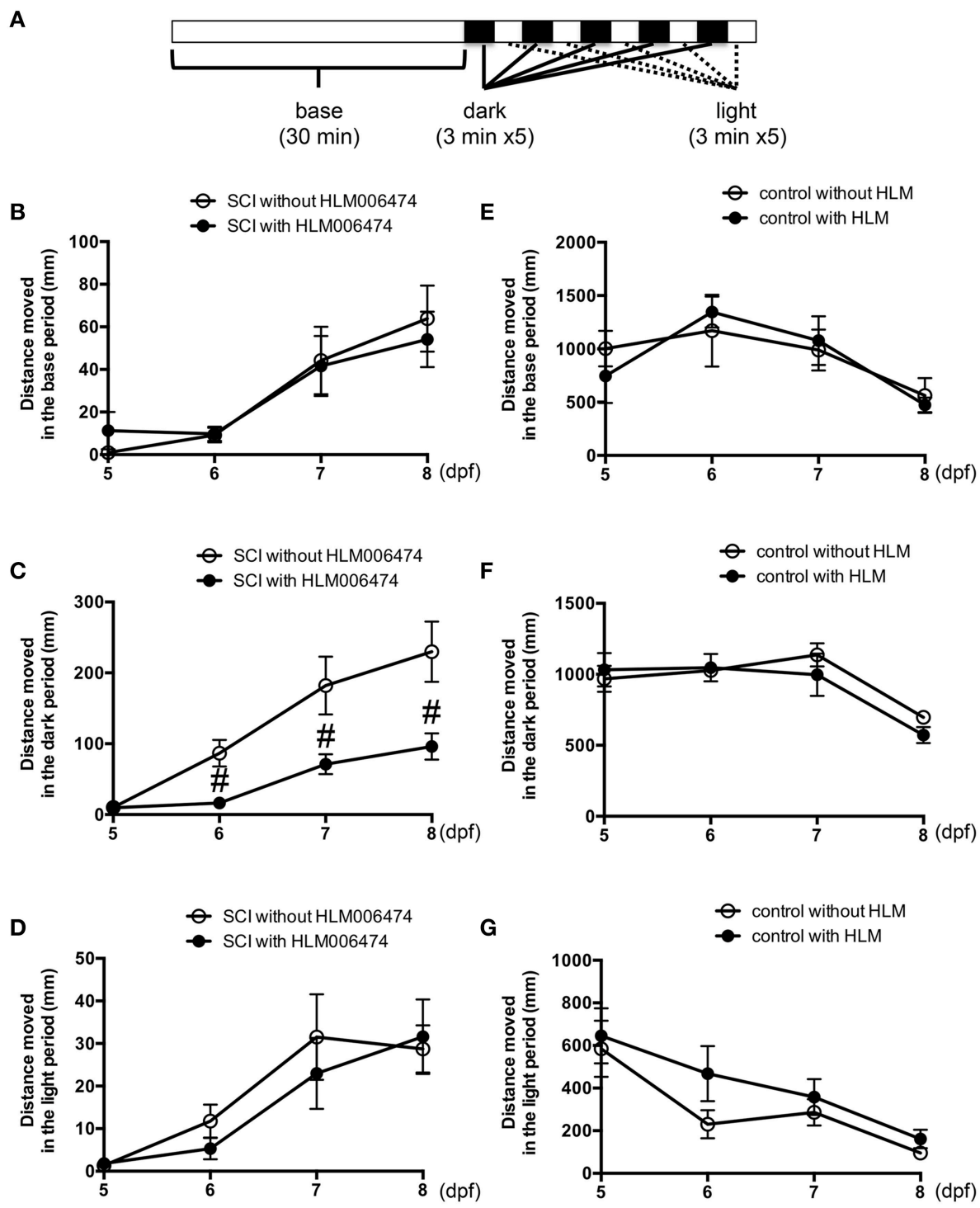

FIGURE 4 | Recovery of zebrafish locomotive behavior after SCI was inhibited by treatment with E2F4 inhibitor. (A) Protocol for the assessment of zebrafish locomotive behavior. Zebrafish were placed in each well of a 48-well-plate. The plate was set in a device to monitor the behavior of zebrafish in real-time. In the device, the plate was illuminated for $30 \mathrm{~min}$ for acclimatization, followed by five sets of dark (3 min) and light (3 min) periods. Zebrafish with SCl (B-D) or without $\mathrm{SCl}(\mathbf{E}-\mathbf{G})$ were treated with an E2F4 inhibitor HLM006474 $(5 \mu \mathrm{M})$ or with vehicle. The distance moved during the base (30 min, B,E), dark periods (15 min, C,F) or light periods (15 min, $\mathbf{D , G}$ ) are shown. $N=36$ for each group with SCl and eight for each group without SCl. $\# p<0.05$ compared to SCl without HLM006474.

when cells exit the cell cycle and enter into G0 phase in response to differentiation signals or absence of growth factors (Sadasivam and DeCaprio, 2013). When cells leave G0 and enter into the cell cycle, RBL2 dissociates from E2F4 and the MuvB core resulting in the release of the DREAM complex from the promoters of genes related to the cell cycle (Sadasivam and DeCaprio, 2013). When cells enter into the G2/M stage, the MuvB core proteins associate with FOXM1, activating the expression of genes related to the G2/M stage, including CCNA2, CCNB2, NEK2, and PLK1 (Teh et al., 2010; Bella et al., 2014). The expression of these genes was down-regulated in zebrafish SCI at 1 and 3 dpi (Tables S1, S2), suggesting that e2f4 and tfdp 1 may be activated and foxm1 may be inhibited after SCI in zebrafish. Genes related to the cell cycle were also significantly enriched in the DEGs specific for zebrafish SCI potentially regulated by at least two of e2f4, tfdp 1 and foxm 1 at 1 and 3 dpi (Tables S5, S6), suggesting important roles of these TFs in the regulation of DEGs specific for zebrafish SCI.

\section{Regulation of Mouse/Rat SCI-Specific DEGs}

In this study, we demonstrated that Nfic and Tead4 may contribute to the regulation of DEGs specific for mouse/rat DEG. We cannot exclude the possibility, however, that other important factors may regulate the DEGs specific for mammalian 
A
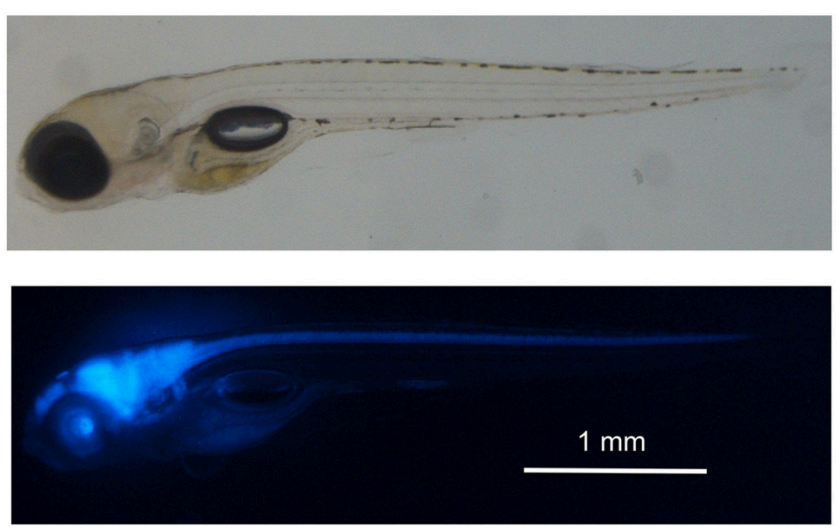

B

SCl without HLM006474

\section{C}
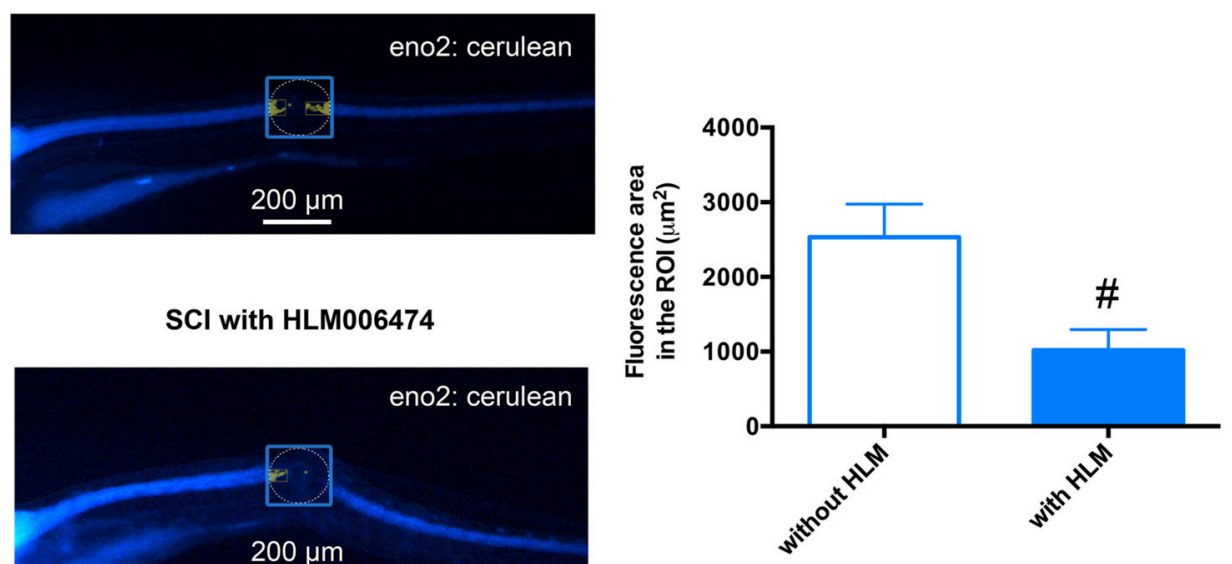

FIGURE 5 | Neuronal regeneration after SCI was impaired in zebrafish treated with E2F4 inhibitor. (A) Representative in vivo imaging of Tg(eno2: Cerulean) zebrafish. (B) Representative in vivo imaging of Tg(eno2: Cerulean) zebrafish with SCI at 3 dpi treated with or without HLM006474. (C) The Cerulean area in the ROI was quantified and compared between zebrafish with SCl treated with or without HLM006474. N=24 and 22 for zebrafish without and with HLM006474, respectively. ${ }^{\#} p<0.05$ compared to SCI without HLM006474.

SCI. It has been demonstrated that the expression of miR$133 \mathrm{~b}$ was increased and decreased after SCI in zebrafish (Yu et al., 2011) and rat (Liu et al., 2009), respectively. Connective tissue growth factor (CTGF) is a target of miR-133, and it is down-regulated by miR-133 (Duisters et al., 2009). Furthermore, the expression of CTGF in reactive astrocytes was significantly increased through the down-regulation of miR133b (Xin et al., 2013), while CTGF expression by reactive astrocytes is associated with matrix deposition and glial scar formation in human cerebral infarction (Schwab et al., 2000). These findings suggest that down-regulation of miR-133b may be responsible, at least in part, for DEGs specific to mammalian SCI. Consistent with this, 19 genes identified as mammalian SCI-specific DEGs at 3 dpi are also predicted to be targets of miR-133b (Wong and Wang, 2015). Further studies are required to elucidate the mechanisms regulating DEGs specific for mammalian SCI.

\section{Activation of E2F4 is Responsible for Significant Recovery after SCl in Zebrafish}

We demonstrated that the recovery of locomotive function and neuronal regeneration after SCI were significantly inhibited in zebrafish treated with HLM00646, an E2F4 inhibitor, suggesting that activation of e2f4 after SCI may be responsible, at least in part, for significant recovery in zebrafish. To our knowledge, E2F4 is the only target of HLM00646; however, we cannot exclude the possibility that HLM00646 inhibited zebrafish SCI recovery through targeting molecules other than e2f4. Experiments using e2f4 knock-down or knock-out zebrafish will confirm the involvement of e2f4 in the significant recovery from SCI in zebrafish. The mechanism of how e2f4 is activated after SCI in zebrafish also remains to be elucidated. It has been demonstrated that E2F4 does not contain a nuclear localization signal, and that the nuclear import of E2F4 is stimulated by association with RBL2 (also called p130) (Moberg et al., 1996). The association 
between E2F4 and RBL2 is stimulated in response to cell cycle exit (Moberg et al., 1996). It has also been demonstrated that transcriptional repression activity of the DREAM complex is regulated by phosphorylation of the MuvB complex (Sadasivam and DeCaprio, 2013). The MuvB complex is phosphorylated by DYRK1A (Litovchick et al., 2011). Transient expression of DYRK1A in neuronal precursors acts as a binary switch, coupling the end of proliferation and the initiation of neuronal differentiation (Hammerle et al., 2011). These findings suggest that the function of e2f 4 may be stimulated by association with rbl2 and activation of dyrk1a after SCI in zebrafish.

The selective activation of e2f4 in zebrafish SCI also suggests that the function of E2F4 may be inhibited in mammalian SCI. It has been demonstrated that 4-hydroxynonenal (HNE), a product of lipid peroxidation, significantly reduced E2F4 protein levels and increased those of the pRb/E2F1 complex (Barrera et al., 2004). E2F4 and E2F1 have opposing roles in regulation of the cell cycle (Crosby and Almasan, 2004). It has also been demonstrated that E2F4 may be transcriptionally repressed by E2F1 (Ma et al., 2004). These findings suggest that the activation of E2F1 may inhibit the function of E2F4 in mammalian SCI. This is consistent with previous reports demonstrating that antioxidant therapies (Bains and Hall, 2012) and inhibition of E2F1 (Wu et al., 2012) confers neuroprotection after SCI in mammals. It has been demonstrated that forced expression of cyclin-dependent kinase inhibitor 1A (CDKN1A) can promote axonal regeneration following SCI in rats (Tanaka et al., 2004). CDKN1A inhibits E2F1 (Ookawa et al., 2001) and activates E2F4 (Benson et al., 2014). These studies suggest that CDKN1A may be inhibited after SCI in mammals, resulting in the activation of E2F1, and inhibition of E2F4. Activation of E2F4 may therefore be a therapeutic strategy after SCI in mammals.

\section{Screening Chemicals and Genes for Treatment of SCI Using Larval Zebrafish}

In this study, we developed a SCI model using larval zebrafish that can be used to assess neuronal regeneration by in vivo imaging and functional recovery by behavioral profiling in multiwell-plates. Zebrafish can absorb a wide range of chemicals from the medium in which they swim, and metabolize them in a similar way to mammals (Nishimura et al., 2015a). Zebrafish are also highly amenable to genome editing (Kotani et al., 2015). Phenotype-based screening using zebrafish has successfully discovered novel therapeutic drugs, novel uses for existing drugs and novel disease-related genes (MacRae and Peterson, 2015). Integration of screening using zebrafish and detailed characterization using rodents may minimize risks in extrapolating animal model data to human biology (Sogorb et al., 2014). Behavioral profiling and in vivo imaging of neuronal cells using larval zebrafish is a useful strategy for identifying genes and chemicals promoting recovery after SCI.

In summary, we demonstrated that activation of e2f4 may be responsible, at least in part, for significant recovery after SCI in zebrafish. This provides novel insights into the mechanism underlying the poor recovery after SCI in mammals, and into potential therapeutic strategies.

\section{AUTHOR CONTRIBUTIONS}

YN and SS are co-first authors. YN conceived the study, performed comparative transcriptome analysis using systems biology approaches, and wrote the paper. SS performed the zebrafish experiments, analyzed the data, and draw the summary illustration. YH performed zebrafish experiments. $\mathrm{SM}, \mathrm{YA}, \mathrm{MY}, \mathrm{SO}, \mathrm{KK}$, and RK provided assistance with experiments. TT conceived the experiments and wrote the paper.

\section{ACKNOWLEDGMENTS}

This work was supported, in part, by the National Institute of Biological Innovation (J14021L025), JSPS KAKENHI (25670127, 15K15051, 24510069), JST A-step (AS262Z00004Q), and Longrange Research Initiative of the Japan Chemical Industrial Association (13_PT01-01). We are grateful to Prof. Kosei Takeuchi (Aichi Medical University) for essential conceptual help in developing the zebrafish SCI model. We also thank Mr. Shinji Umezawa (Sophia Scientific) for his excellent advice on the use of DanioVision and EthoVision. We gratefully acknowledge Junko Koiwa, Hiroko Nakayama, Yuka Takahashi, Chizuru Hirota, and Michiko Ariyoshi for assistance with experiments, and Rie Ikeyama and Yuka Mizutani for secretarial support.

\section{SUPPLEMENTARY MATERIAL}

The Supplementary Material for this article can be found online at: http://journal.frontiersin.org/article/10.3389/fphar. 2016.00119

Table S1 | DEGs in each SCI model and the overlap at 1 dpi. S1-1, DEGs in mouse SCl at 1 dpi; $\mathbf{S 1 - 2}$, DEGs in rat SCl at 1 dpi; $\mathbf{S 1 - 3}$, DEGs in zebrafish SCl at $1 \mathrm{dpi}$; $\mathbf{S 1 - 4}$, DEGs common among mouse, rat, and zebrafish $\mathrm{SCl}$ at $1 \mathrm{dpi}$; S1-5, DEGs showing opposite differential expression between zebrafish and mouse/rat SCl at 1 dpi; $\mathbf{S 1 - 6 , ~ D E G s ~ u n i q u e ~ i n ~ z e b r a f i s h ~ S C l ~ a t ~} 1$ dpi; $\mathbf{S 1 - 7}$, DEGs unique in mouse and rat $\mathrm{SCl}$ at $1 \mathrm{dpi}$; $\mathbf{S 1 - 8}$, DEGs specific for zebrafish $\mathrm{SCl}$ at 1 dpi; S1-9, DEGs specific for mouse/rat SCl at 1 dpi.

Table S2 | DEGs in each SCI model and the overlap at 3 dpi. S2-1, DEGs in mouse SCl at 3 dpi; $\mathbf{S 2 - 2}$, DEGs in rat SCl at 3 dpi; $\mathbf{S 2 - 3}$, DEGs in zebrafish SCl at 3 dpi; S2-4, DEGs common among mouse, rat, and zebrafish SCl at 3 dpi; S2-5, DEGs showing opposite differential expression between zebrafish and mouse/rat SCl at 3 dpi; S2-6, DEGs unique in zebrafish SCl at 3 dpi; $\mathbf{S 2 - 7}$, DEGs unique in mouse and rat $\mathrm{SCl}$ at $3 \mathrm{dpi}$; $\mathbf{S 2 - 8}$, DEGs specific for zebrafish $\mathrm{SCl}$ at 3 dpi; S2-9, DEGs specific for mouse/rat SCl at 3 dpi.

Table S3 | Gene ontologies enriched in DEGs specific for zebrafish or mouse/rat SCI. S3-1 Gene ontologies enriched in DEGs specific for zebrafish $\mathrm{SCl}$ at $1 \mathrm{dpi}$; S3-2, Gene ontologies enriched in DEGs specific for zebrafish $\mathrm{SCl}$ at 3 dpi; S3-3, Gene ontologies enriched in DEGs specific for mouse/rat SCl at 1 dpi; S3-4, Gene ontologies enriched in DEGs specific for mouse/rat SCl at 3 dpi.

Table S4 | Transcription factors potentially regulating DEGs specific for zebrafish SCI or mouse/rat SCI. S4-1, Transcription factors potentially regulating DEGs specific for zebrafish SCl; S4-2, Transcription factors potentially regulating DEGs specific for mouse/rat $\mathrm{SCl}$.

Table S5 | DEGs specific for zebrafish or mouse/rat SCI potentially regulated by TFs. S5-1 DEGs specific for zebrafish $\mathrm{SCl}$ at 1 dpi potentially regulated by at least two of e2f4, tfdp1, and foxm1; S5-2, DEGs specific for zebrafish $\mathrm{SCl}$ at 3 dpi potentially regulated by at least two of e2f4, tfdp1, and 
foxm1; S5-3, DEGs specific for mouse/rat SCl at 1 dpi potentially regulated by Nfic and Tead4; S5-4, DEGs specific for mouse/rat SCl at 3 dpi potentially regulated by Nfic and Tead4.

Table S6 | Gene ontologies enriched in DEGs potentially regulated by TFs. s6-1 Gene ontologies enriched in DEGs specific for zebrafish SCl at $1 \mathrm{dpi}$ potentially regulated by at least two of e2f4, tfdp1, and foxm1; S6-2, Gene ontologies enriched in DEGs specific for zebrafish $\mathrm{SCl}$ at 3 dpi potentially regulated by at least two of e2f4, tfdp1, and foxm1; $\mathbf{S 6 - 3}$, Gene ontologies

\section{REFERENCES}

Bai, Q., Garver, J. A., Hukriede, N. A., and Burton, E. A. (2007). Generation of a transgenic zebrafish model of Tauopathy using a novel promoter element derived from the zebrafish eno2 gene. Nucleic Acids Res. 35, 6501-6516. doi: 10.1093/nar/gkm608

Bains, M., and Hall, E. D. (2012). Antioxidant therapies in traumatic brain and spinal cord injury. Biochim. Biophys. Acta 1822, 675-684. doi: 10.1016/j.bbadis.2011.10.017

Barrera, G., Pizzimenti, S., and Dianzani, M. U. (2004). 4-hydroxynonenal and regulation of cell cycle: effects on the pRb/E2F pathway. Free Radic. Biol. Med. 37, 597-606. doi: 10.1016/j.freeradbiomed.2004.05.023

Barrett, T., Troup, D. B., Wilhite, S. E., Ledoux, P., Rudnev, D., Evangelista, C., et al. (2009). NCBI GEO: archive for high-throughput functional genomic data. Nucleic Acids Res. 37, D885-D890. doi: 10.1093/nar/gkn764

Bella, L., Zona, S., Nestal De Moraes, G., and Lam, E. W. (2014). FOXM1: a key oncofoetal transcription factor in health and disease. Semin. Cancer Biol. 29, 32-39. doi: 10.1016/j.semcancer.2014.07.008

Benson, E. K., Mungamuri, S. K., Attie, O., Kracikova, M., Sachidanandam, R., Manfredi, J. J., et al. (2014). p53-dependent gene repression through p21 is mediated by recruitment of E2F4 repression complexes. Oncogene 33, 3959-3969. doi: 10.1038/onc.2013.378

Busch, S. A., and Silver, J. (2007). The role of extracellular matrix in CNS regeneration. Curr. Opin. Neurobiol. 17, 120-127. doi: 10.1016/j.conb.2006.09.004

Chamankhah, M., Eftekharpour, E., Karimi-Abdolrezaee, S., Boutros, P. C., SanMarina, S., and Fehlings, M. G. (2013). Genome-wide gene expression profiling of stress response in a spinal cord clip compression injury model. BMC Genomics 14:583. doi: 10.1186/1471-2164-14-583

Chen, G., Fang, X., and Yu, M. (2015). Regulation of gene expression in rats with spinal cord injury based on microarray data. Mol. Med. Rep. 12, 2465-2472. doi: $10.3892 / \mathrm{mmr} .2015 .3670$

Chen, K., Deng, S., Lu, H., Zheng, Y., Yang, G., Kim, D., et al. (2013). RNA-seq characterization of spinal cord injury transcriptome in acute/subacute phases: a resource for understanding the pathology at the systems level. PLoS ONE 8:e72567. doi: 10.1371/journal.pone.0072567

Cowley, M. J., Pinese, M., Kassahn, K. S., Waddell, N., Pearson, J. V., Grimmond, S. M., et al. (2012). PINA v2.0: mining interactome modules. Nucleic Acids Res. 40, D862-D865. doi: 10.1093/nar/gkr967

Crosby, M. E., and Almasan, A. (2004). Opposing roles of E2Fs in cell proliferation and death. Cancer Biol. Ther. 3, 1208-1211. doi: 10.4161/cbt.3.12.1494

Di Giovanni, S., Knoblach, S. M., Brandoli, C., Aden, S. A., Hoffman, E. P., and Faden, A. I. (2003). Gene profiling in spinal cord injury shows role of cell cycle in neuronal death. Ann. Neurol. 53, 454-468. doi: 10.1002/ana.10472

Duisters, R. F., Tijsen, A. J., Schroen, B., Leenders, J. J., Lentink, V., Van Der Made, I., et al. (2009). miR-133 and miR-30 regulate connective tissue growth factor: implications for a role of microRNAs in myocardial matrix remodeling. Circ. Res. 104, 170-178, 176p following 178. doi: 10.1161/circresaha.108.182535

Gautier, L., Cope, L., Bolstad, B. M., and Irizarry, R. A. (2004). affy-analysis of Affymetrix GeneChip data at the probe level. Bioinformatics 20, 307-315. doi: 10.1093/bioinformatics/btg405

Gentleman, R. C., Carey, V. J., Bates, D. M., Bolstad, B., Dettling, M., Dudoit, S., et al. (2004). Bioconductor: open software development for computational biology and bioinformatics. Genome Biol. 5:R80. doi: 10.1186/gb-2004-5-10-r80

Gerstein, M. B., Kundaje, A., Hariharan, M., Landt, S. G., Yan, K. K., Cheng, C., et al. (2012). Architecture of the human regulatory network derived from ENCODE data. Nature 489, 91-100. doi: 10.1038/nature11245 enriched in DEGs specific for mouse/rat $\mathrm{SCl}$ at 1 dpi potentially regulated by Nfic and Tead4; S6-4, Gene ontologies enriched in DEGs specific for mouse/rat SCl at 3 dpi potentially regulated by Nfic and Tead4.

\section{Movie S1 | Cutting the zebrafish spinal cord using a microdissection device.}

Movie S2 | Locomotion of zebrafish with SCI responding to the change from light to dark conditions.

Hammerle, B., Ulin, E., Guimera, J., Becker, W., Guillemot, F., and Tejedor, F. J. (2011). Transient expression of Mnb/Dyrk1a couples cell cycle exit and differentiation of neuronal precursors by inducing p27KIP1 expression and suppressing NOTCH signaling. Development 138, 2543-2554. doi: 10.1242/dev.066167

Hong, F., Breitling, R., Mcentee, C. W., Wittner, B. S., Nemhauser, J. L., and Chory, J. (2006). RankProd: a bioconductor package for detecting differentially expressed genes in meta-analysis. Bioinformatics 22, 2825-2827. doi: 10.1093/bioinformatics/btl476

Huang Da, W., Sherman, B. T., and Lempicki, R. A. (2009). Bioinformatics enrichment tools: paths toward the comprehensive functional analysis of large gene lists. Nucleic Acids Res. 37, 1-13. doi: 10.1093/nar/gkn923

Hui, S. P., Sengupta, D., Lee, S. G. P., Sen, T., Kundu, S., Mathavan, S., et al. (2014). Genome wide expression profiling during spinal cord regeneration identifies comprehensive cellular responses in Zebrafish. PLoS ONE 9:e84212. doi: 10.1371/journal.pone.0084212

Jacquemin, P., Hwang, J. J., Martial, J. A., Dolle, P., and Davidson, I. (1996). A novel family of developmentally regulated mammalian transcription factors containing the TEA/ATTS DNA binding domain. J. Biol. Chem. 271, 21775-21785. doi: 10.1074/jbc.271.36.21775

Janky, R., Verfaillie, A., Imrichova, H., Van De Sande, B., Standaert, L., Christiaens, V., et al. (2014). iRegulon: from a gene list to a gene regulatory network using large motif and track collections. PLoS Comput. Biol. 10:e1003731. doi: 10.1371/journal.pcbi.1003731

Kawakami, K. (2007). Tol2: a versatile gene transfer vector in vertebrates. Genome Biol. 8(Suppl. 1):S7. doi: 10.1186/gb-2007-8-s1-s7

Kelsh, R. N., Brand, M., Jiang, Y. J., Heisenberg, C. P., Lin, S., Haffter, P., et al. (1996). Zebrafish pigmentation mutations and the processes of neural crest development. Development 123, 369-389.

Kotani, H., Taimatsu, K., Ohga, R., Ota, S., and Kawahara, A. (2015). Efficient multiple genome modifications induced by the crRNAs, tracrRNA and Cas9 protein complex in Zebrafish. PLoS ONE 10:e0128319. doi: 10.1371/journal.pone.0128319

Lee-Liu, D., Edwards-Faret, G., Tapia, V. S., and Larrain, J. (2013). Spinal cord regeneration: lessons for mammals from non-mammalian vertebrates. Genesis 51, 529-544. doi: 10.1002/dvg.22406

Lee-Liu, D., Moreno, M., Almonacid, L. I., Tapia, V. S., Munoz, R., Von Marees, J., et al. (2014). Genome-wide expression profile of the response to spinal cord injury in Xenopus laevis reveals extensive differences between regenerative and non-regenerative stages. Neural Dev. 9:12. doi: 10.1186/1749-8104-9-12

Litovchick, L., Florens, L. A., Swanson, S. K., Washburn, M. P., and Decaprio, J. A. (2011). DYRK1A protein kinase promotes quiescence and senescence through DREAM complex assembly. Genes Dev. 25, 801-813. doi: 10.1101/gad.2034211

Liu, N. K., Wang, X. F., Lu, Q. B., and Xu, X. M. (2009). Altered microRNA expression following traumatic spinal cord injury. Exp. Neurol. 219, 424-429. doi: 10.1016/j.expneurol.2009.06.015

Ma, L., Yu, Y. M., Guo, Y., Hart, R. P., and Schachner, M. (2012). Cysteine- and glycine-rich protein 1a is involved in spinal cord regeneration in adult zebrafish. Eur. J. Neurosci. 35, 353-365. doi: 10.1111/j.1460-9568.2011.07958.x

Ma, Y., Freeman, S. N., and Cress, W. D. (2004). E2F4 deficiency promotes druginduced apoptosis. Cancer Biol. Ther. 3, 1262-1269. doi: 10.4161/cbt.3.12.1239

Ma, Y., Kurtyka, C. A., Boyapalle, S., Sung, S. S., Lawrence, H., Guida, W., et al. (2008). A small-molecule E2F inhibitor blocks growth in a melanoma culture model. Cancer Res. 68, 6292-6299. doi: 10.1158/0008-5472.CAN-08-0121

MacPhail, R. C., Brooks, J., Hunter, D. L., Padnos, B., Irons, T. D., and Padilla, S. (2009). Locomotion in larval zebrafish: influence of time of day, lighting and ethanol. Neurotoxicology 30, 52-58. doi: 10.1016/j.neuro.2008.09.011 
MacRae, C. A., and Peterson, R. T. (2015). Zebrafish as tools for drug discovery. Nat. Rev. Drug Discov. 14, 721-731. doi: 10.1038/nrd4627

Moberg, K., Starz, M. A., and Lees, J. A. (1996). E2F-4 switches from p130 to p107 and pRB in response to cell cycle reentry. Mol. Cell. Biol. 16, 1436-1449. doi: 10.1128/MCB.16.4.1436

Nishimura, Y., Inoue, A., Sasagawa, S., Koiwa, J., Kawaguchi, K., Kawase, R., et al. (2016). Using zebrafish in systems toxicology for developmental toxicity testing. Congenit. Anom. (Kyoto). 56, 18-27. doi: 10.1111/cga.12142

Nishimura, Y., Murakami, S., Ashikawa, Y., Sasagawa, S., Umemoto, N., Shimada, Y., et al. (2015a). Zebrafish as a systems toxicology model for developmental neurotoxicity testing. Congenit. Anom. (Kyoto). 55, 1-16. doi: $10.1111 /$ cga. 12079

Nishimura, Y., Okabe, S., Sasagawa, S., Murakami, S., Ashikawa, Y., Yuge, M., et al. (2015b). Pharmacological profiling of zebrafish behavior using chemical and genetic classification of sleep-wake modifiers. Front. Pharmacol 6:257. doi: 10.3389/fphar.2015.00257

Nishimura, Y., Sasagawa, S., Ariyoshi, M., Ichikawa, S., Shimada, Y., Kawaguchi, K., et al. (2015c). Systems pharmacology of adiposity reveals inhibition of EP300 as a common therapeutic mechanism of caloric restriction and resveratrol for obesity. Front. Pharmacol. 6:199. doi: 10.3389/fphar.2015.00199

Ookawa, K., Tsuchida, S., Kohno, T., and Yokota, J. (2001). Alterations in expression of E2F-1 and E2F-responsive genes by RB, p53 and p21(Sdi1/WAF1/Cip1) expression. FEBS Lett. 500, 25-30. doi: 10.1016/S00145793(01)02583-2

Pjanic, M., Pjanic, P., Schmid, C., Ambrosini, G., Gaussin, A., Plasari, G., et al. (2011). Nuclear factor I revealed as family of promoter binding transcription activators. BMC Genomics 12:181. doi: 10.1186/1471-2164-12-181

Ritchie, M. E., Phipson, B., Wu, D., Hu, Y., Law, C. W., Shi, W., et al. (2015). Limma powers differential expression analyses for RNA-sequencing and microarray studies. Nucleic Acids Res. 43, e47. doi: 10.1093/nar/gkv007

Sadasivam, S., and DeCaprio, J. A. (2013). The DREAM complex: master coordinator of cell cycle-dependent gene expression. Nat. Rev. Cancer 13, 585-595. doi: 10.1038/nrc3556

Sasagawa, S., Nishimura, Y., Kon, T., Yamanaka, Y., Murakami, S., Ashikawa, Y., et al. (2016). DNA damage response is involved in the developmental toxicity of mebendazole in zebrafish retina. Front. Pharmacol. 7:57. doi: 10.3389/fphar.2016.00057

Schwab, J. M., Postler, E., Nguyen, T. D., Mittelbronn, M., Meyermann, R., and Schluesener, H. J. (2000). Connective tissue growth factor is expressed by a subset of reactive astrocytes in human cerebral infarction. Neuropathol. Appl. Neurobiol. 26, 434-440. doi: 10.1046/j.1365-2990.2000.00271.x

Shannon, P., Markiel, A., Ozier, O., Baliga, N. S., Wang, J. T., Ramage, D., et al. (2003). Cytoscape: a software environment for integrated models of biomolecular interaction networks. Genome Res. 13, 2498-2504. doi: $10.1101 /$ gr. 1239303

Silver, J., Schwab, M. E., and Popovich, P. G. (2015). Central nervous system regenerative failure: role of oligodendrocytes, astrocytes, and microglia. Cold Spring Harb. Perspect. Biol. 7:a020602. doi: 10.1101/cshperspect.a020602

Smedley, D., Haider, S., Durinck, S., Pandini, L., Provero, P., Allen, J., et al. (2015). The BioMart community portal: an innovative alternative to large, centralized data repositories. Nucleic Acids Res. 43, W589-W598. doi: 10.1093/nar/gkv350

Sogorb, M. A., Pamies, D., De Lapuente, J., Estevan, C., Estevez, J., and Vilanova, E. (2014). An integrated approach for detecting embryotoxicity and developmental toxicity of environmental contaminants using in vitro alternative methods. Toxicol. Lett. 230, 356-367. doi: 10.1016/j.toxlet.2014.01.037
Tanaka, H., Yamashita, T., Yachi, K., Fujiwara, T., Yoshikawa, H., and Tohyama, M. (2004). Cytoplasmic p21(Cip1/WAF1) enhances axonal regeneration and functional recovery after spinal cord injury in rats. Neuroscience 127, 155-164. doi: 10.1016/j.neuroscience.2004.05.010

Teh, M. T., Gemenetzidis, E., Chaplin, T., Young, B. D., and Philpott, M. P. (2010). Upregulation of FOXM1 induces genomic instability in human epidermal keratinocytes. Mol. Cancer 9:45. doi: 10.1186/14764598-9-45

Tian, D. S., Xie, M. J., Yu, Z. Y., Zhang, Q., Wang, Y. H., Chen, B., et al. (2007). Cell cycle inhibition attenuates microglia induced inflammatory response and alleviates neuronal cell death after spinal cord injury in rats. Brain Res. 1135, 177-185. doi: 10.1016/j.brainres.2006.11.085

Vajn, K., Plunkett, J. A., Tapanes-Castillo, A., and Oudega, M. (2013). Axonal regeneration after spinal cord injury in zebrafish and mammals: differences, similarities, translation. Neurosci. Bull. 29, 402-410. doi: 10.1007/s12264-0131361-8

Watanabe, K., Nishimura, Y., Nomoto, T., Umemoto, N., Zhang, Z., Zhang, B., et al. (2012). In vivo assessment of the permeability of the blood-brain barrier and blood-retinal barrier to fluorescent indoline derivatives in zebrafish. $B M C$ Neurosci. 13:101. doi: 10.1186/1471-2202-13-101

Westerfield, M. (2007). A Guide for the Laboratory Use of Zebrafish (Danio rerio). Eugene, OR: University of Oregon Press.

Wong, N., and Wang, X. (2015). miRDB: an online resource for microRNA target prediction and functional annotations. Nucleic Acids Res. 43, D146-D152. doi: 10.1093/nar/gku1104

Wu, J., Kharebava, G., Piao, C., Stoica, B. A., Dinizo, M., Sabirzhanov, B., et al. (2012). Inhibition of E2F1/CDK1 pathway attenuates neuronal apoptosis in vitro and confers neuroprotection after spinal cord injury in vivo. PLOS ONE 7:e42129. doi: 10.1371/journal.pone.0042129

Wu, J., Renn, C. L., Faden, A. I., and Dorsey, S. G. (2013). TrkB.T1 contributes to neuropathic pain after spinal cord injury through regulation of cell cycle pathways. J. Neurosci. 33, 12447-12463. doi: 10.1523/JNEUROSCI.084613.2013

Wu, J., Stoica, B. A., and Faden, A. I. (2011). Cell cycle activation and spinal cord injury. Neurotherapeutics 8, 221-228. doi: 10.1007/s13311-011-0028-2

Xin, H., Li, Y., Liu, Z., Wang, X., Shang, X., Cui, Y., et al. (2013). MiR$133 \mathrm{~b}$ promotes neural plasticity and functional recovery after treatment of stroke with multipotent mesenchymal stromal cells in rats via transfer of exosome-enriched extracellular particles. Stem Cells 31, 2737-2746. doi: 10.1002/stem.1409

Yu, Y. M., Gibbs, K. M., Davila, J., Campbell, N., Sung, S., Todorova, T. I., et al. (2011). MicroRNA miR-133b is essential for functional recovery after spinal cord injury in adult zebrafish. Eur. J. Neurosci. 33, 1587-1597. doi: $10.1111 / j .1460-9568.2011 .07643 . x$

Conflict of Interest Statement: The authors declare that the research was conducted in the absence of any commercial or financial relationships that could be construed as a potential conflict of interest.

Copyright $\odot 2016$ Sasagawa, Nishimura, Hayakawa, Murakami, Ashikawa, Yuge, Okabe, Kawaguchi, Kawase and Tanaka. This is an open-access article distributed under the terms of the Creative Commons Attribution License (CC BY). The use, distribution or reproduction in other forums is permitted, provided the original author(s) or licensor are credited and that the original publication in this journal is cited, in accordance with accepted academic practice. No use, distribution or reproduction is permitted which does not comply with these terms. 